\title{
Acute presentation of the pregnant patient
}

\author{
Authors: Francesca Neuberger ${ }^{A}$ and Catherine Nelson-Piercy ${ }^{B}$
}

\section{Three-quarters of maternal deaths are in women with coexisting medical complications. It can be challenging to differentiate symptoms of normal pregnancy from pathological symptomatology, and physicians need to be mindful of special considerations in assessing and managing acute medical problems in pregnancy. This article focuses on women presenting with shortness of breath, chest pain and palpitations.}

\section{Introduction}

Physicians participating in the acute medical take are likely to encounter pregnant women on the acute medical unit, or may be asked to give an opinion on the obstetric ward. Pregnancy does not usually alter acute presentations but there are some special considerations in clinical assessment and differential diagnosis.

The MBRACCE report into UK maternal deaths from 2009-2012 $2^{1}$ showed a decrease in mortality from obstetric causes. However, mortality rates from indirect causes (medical and psychiatric) have not altered over the past ten years. Nearly three-quarters of all women who died had a coexisting medical complication. The report recommends that physicians are appropriately trained in the care of pregnant women, and that women with medical disorders in pregnancy have access to coordinated obstetric and medical input.

This review discusses the approach to pregnant women presenting with common symptoms of shortness of breath, chest pain and palpitations.

\section{Shortness of breath}

A degree of breathlessness is a feature of normal pregnancy. The challenge for the clinician is identifying pathological symptomatology (Fig 1). From the history, women with physiological breathlessness of pregnancy typically describe an 'air hunger' at rest and particularly when talking, which may be relieved by activity.

In the examination, features such as tachycardia may be within normal limits (see Box 1). A useful bedside test is to

Authors: Aregistrar in acute and general medicine and obstetric medicine, Guy's and St Thomas' Foundation Trust, London, UK; Bprofessor of obstetric medicine and consultant obstetric physician, Guy's and St Thomas' Foundation Trust and Imperial College Health Care Trust, London, UK monitor oxygen saturations with a pulse oximeter on exercise, for example climbing stairs. This allows the clinician to evaluate the degree of breathlessness with gentle exertion, and monitor oxygen saturations. While there is no widely accepted formal protocol for this, it is a reassuring feature if oxygen saturations do not decrease on exercise.

Normal results of commonly requested investigations in pregnancy are detailed in Table 1. Asthma is common and has a variable course in pregnancy. Acute severe asthma should be managed vigorously with the same drug treatments, including magnesium, steroids and theophyllines, as outside of pregnancy. There are extensive safety data for all these drugs in pregnancy, and guidelines stress the importance of not withholding steroids, in particular, ${ }^{5}$ chest X-rays (CXR) should not be withheld where indicated. Continuous fetal monitoring should be used for women over 24 -weeks gestation with acute severe asthma. Intensivists should be involved early. Acute asthma attacks are very rare in labour, perhaps due to higher circulating levels of endogenous steroids. ${ }^{5}$

Bacterial pneumonia is no more common in pregnancy, but there is an increased susceptibility to viral pneumonias including Varicella zoster and influenza. CXR should not be withheld if pneumonia is suspected, and treatment with antivirals should be initiated for women with pneumonia secondary to flu viruses. The flu vaccine is recommended for all pregnant women. In the most recent MBRACCE report, influenza was the cause of death in 1 in 11 maternal mortalities. More than half of these deaths could have been prevented by vaccination.

Pulmonary embolism (PE) remains the commonest direct cause of maternal mortality and should be suspected in women with sudden-onset breathlessness and pleuritic pain or collapse. It should be investigated, as outlined in Fig 2. It is notoriously difficult to diagnose or exclude on clinical grounds alone, and many women will undergo imaging. The prevalence of ultimately diagnosed PE in pregnant women undergoing imaging for suspected PE is low at $1.4-4.2 \%{ }^{7} \mathrm{D}$-dimer is not validated in pregnancy, ${ }^{6}$ therefore it is unhelpful in enabling clinicians to target their imaging appropriately. However, the presence of risk factors is helpful in risk stratifying pregnant women with suspected PE. Those with pre-existing risk factors (age $>35$ years, raised body mass index, previous venous thromboembolism (VTE), varicose veins, cardiac disease or recent hospital admission) and pregnancy-related risk factors (multiparity, in vitro fertilisation, pre-eclampsia, antenatal/ postpartum haemorrhage, caesarean section or hyperemesis gravidarum) are more likely to develop VTE in pregnancy or postpartum. ${ }^{7}$ A V/Q scan should be requested in preference to a computed tomography pulmonary angiography (CTPA) 


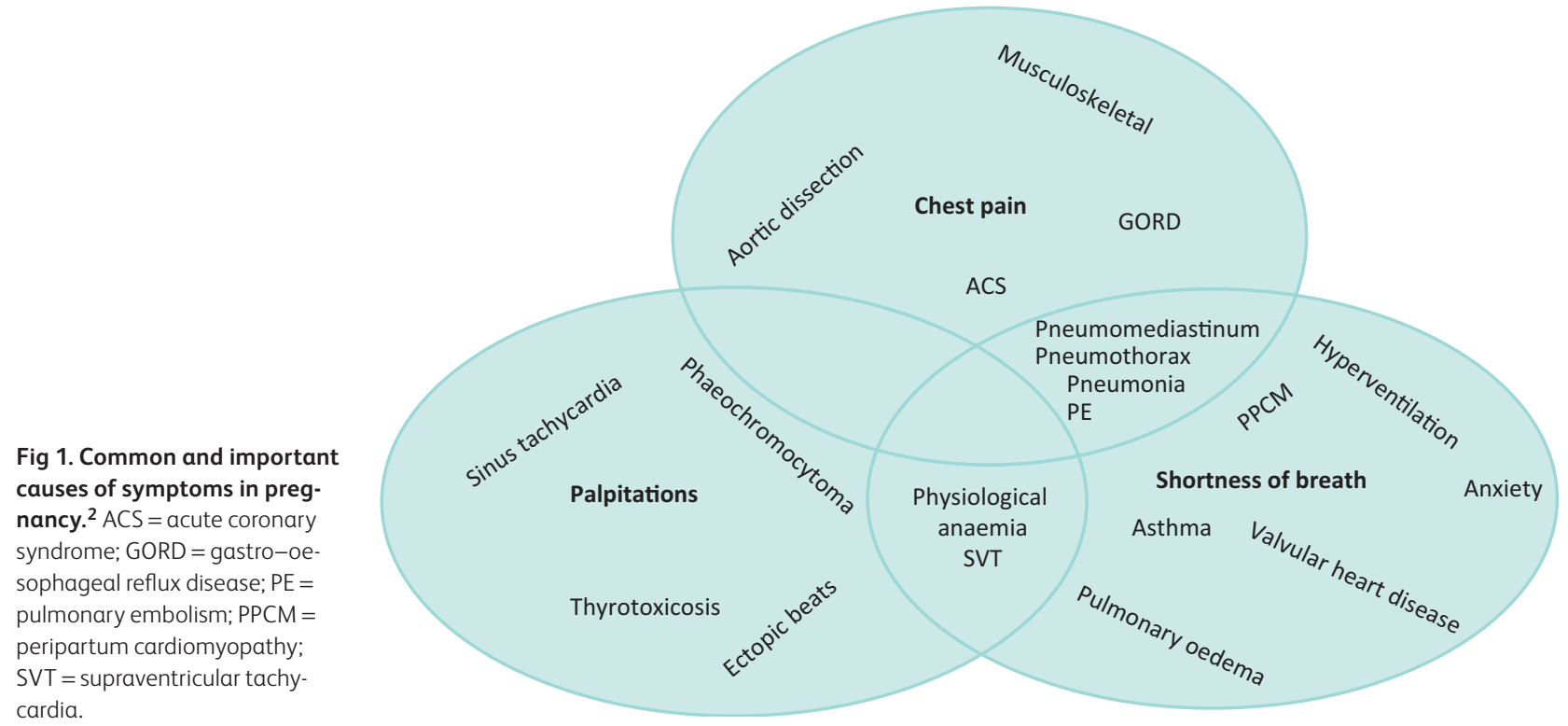

in women with a normal CXR, because the radiation dose to maternal lung and breast is reduced. The fetal radiation exposure associated with CTPA and V/Q is approximately 0.1 $\mathrm{mGy}$ and $0.5 \mathrm{mGy}$ respectively, although quoted figures vary depending on the imaging protocol used. ${ }^{6}$ These doses are well below the $50 \mathrm{mGy}$ maximum recommended exposure in pregnancy. ${ }^{2}$

In women diagnosed with massive PE in pregnancy, intravenous unfractionated heparin is the first-line treatment of choice. In those with massive PE associated with circulatory collapse and risk of imminent arrest, thrombolysis should be considered. It is potentially life-saving, and should not be withheld. Thrombolysis is increasingly used for submassive PE with high clot burden to reduce the risk of chronic pulmonary hypertension. There is no increased risk of haemorrhage

\section{Key points}

Symptoms of normal pregnancy can be difficult to differentiate from symptoms secondary to medical problems, and a careful history, examination and selected investigations are required

Three-quarters of maternal deaths are in women with coexisting medical complications

The vast majority of investigations and treatments should not be withheld in pregnancy

Women with medical problems in pregnancy should be managed with a multidisciplinary team approach

KEYWORDS: Pregnancy, acute medicine, shortness of breath, chest pain, palpitations compared with outside pregnancy. ${ }^{8}$ The approach to thrombolysing a pregnant woman should be multidisciplinary, with involvement of obstetrics, intensivists, experienced physicians and radiologists. Alternative therapeutic options used successfully in pregnancy include catheter-directed thrombolytic therapy, or thoracotomy and surgical embolectomy.

An echocardiogram is key in diagnosing valvular heart disease. While many women will have pre-existing diagnoses, they may only become symptomatic in the latter stages of pregnancy. First presentation of valvular heart disease is seen most in the migrant population. Women have a poorer pregnancy outcome if they fall into New York Heart Association class III and IV, regardless of the nature of the lesion. ${ }^{2}$ Women with regurgitant lesions and normal left ventricular function are low risk. Those with stenotic lesions and impaired left ventricular function are higher risk. Women with pre-existing heart disease should be offered pre-pregnancy counseling and be monitored closely

\section{Box 1. Normal cardiorespiratory examination}

\section{findings in pregnancy.}

> Blood pressure falls by $10 \mathrm{mmHg}$ by $20 / 40$ then returns to prepregnancy level by term

$>$ Respiratory rate may increase marginally. Respiratory rate $>20$ breaths per minute is tachypnoeic

$>$ No significant increase in temperature

> Pulse oximetry is unchanged

> Pulse increases by $10-20$ bpm by third trimester and can be bounding

> Ejection systolic murmur audible throughout praecordium (without carotid radiation)

$>$ Third heart sound

Peripheral oedema 


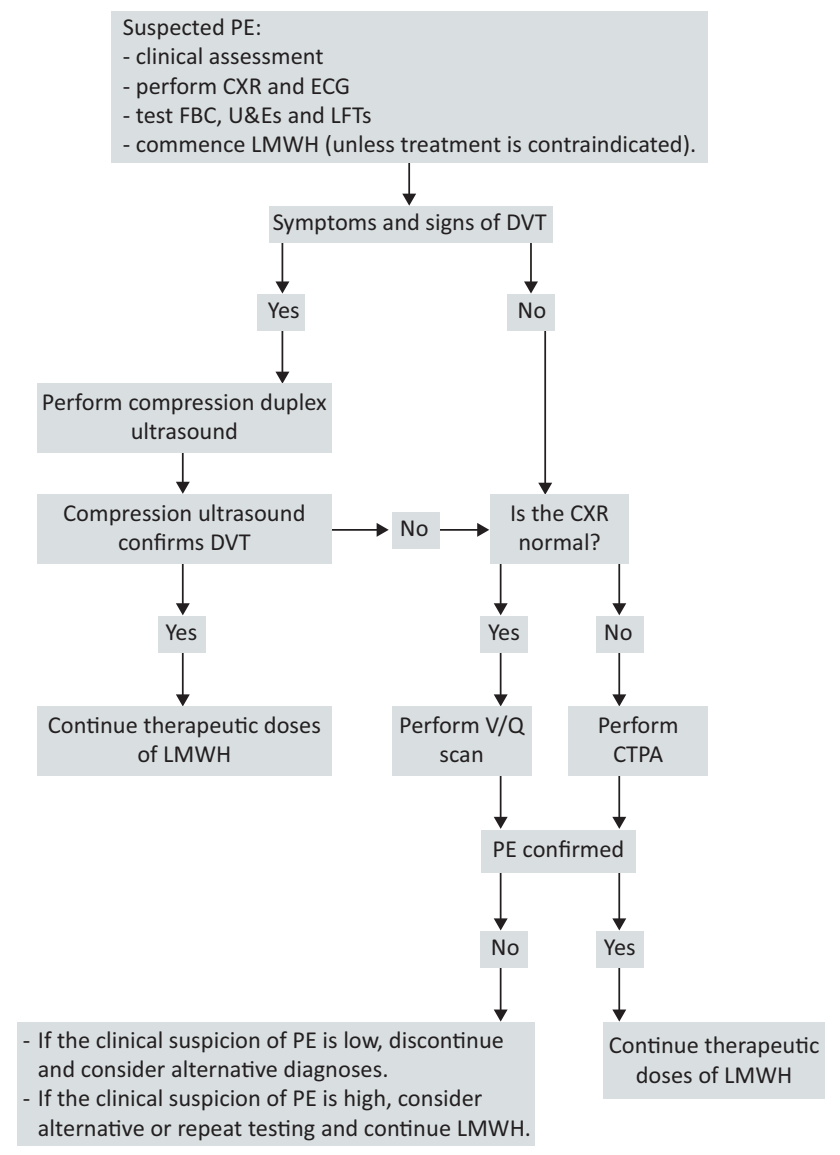

Fig 2. Algorithm for the investigation and initial management of suspected PE in pregnancy and the puerperium. Reproduced with permission. ${ }^{6}$ CTPA = computerised tomography pulmonary angiogram; $\mathrm{CXR}=$ chest $\mathrm{X}$-ray; $\mathrm{DVT}=$ deep vein thrombosis; $\mathrm{ECG}=$ electrocardiogram; $F B C=$ full blood count; $\mathrm{LFT}=$ liver function test; $\mathrm{LMWH}=$ low-molecularweight heparin; $\mathrm{PE}=$ pulmonary embolism; $\mathrm{U \& E}=$ urea and electrolytes.

through their pregnancy. Those with mechanical heart valves need expert management of their anticoagulation.

Pregnant women are more susceptible to pulmonary oedema. Clinicians need to look for and address underlying causes such as underlying cardiac disease, pregnancy-induced hypertension and fluid overload. ${ }^{9}$

\section{Chest pain}

Chest pain is not part of normal pregnancy, although symptoms such as ankle swelling, breathlessness and tachycardia may be normal. The differential diagnosis for chest pain in pregnancy is outlined in Table 1 .

On examination, many healthy pregnant women will have ejection systolic murmurs (see Box 1). Diastolic murmurs should always be considered pathological. The interpretation of common investigations for chest pain in pregnancy is outlined in Table 1.

Gastro-oesophageal reflux is diagnosed on the basis of the history and is very common, affecting two-thirds of women, particularly in the third trimester. It is due to the effect of progesterone on the lower oesophageal sphincter and the
Table 1. Normal findings in pregnancy for common

investigations for breathlessness, chest pain and

palpitations. 3.4

\begin{tabular}{|c|c|}
\hline Investigation & Normal finding in pregnancy \\
\hline $\mathrm{FBC}$ & $\begin{array}{l}\text { Normal } \mathrm{Hb} \text { reference range altered in } \\
\text { pregnancy }(\mathrm{Hb} 105-140 \mathrm{~g} / \mathrm{L}) \\
\text { Normal WBC elevated }\left(6-16 \times 10^{9} / \mathrm{L}\right)\end{array}$ \\
\hline Renal profile & $\begin{array}{l}\text { Increased glomerular filtration rate } \\
\text { Creatinine falls in first and second trimester } \\
\text { Normal urea reference range } 2.5-4.0 \mathrm{mmol} / \mathrm{L} \\
\text { Normal creatinine } 40-80 \mu \mathrm{mol} / \mathrm{L}\end{array}$ \\
\hline Troponin I or T & $\begin{array}{l}\text { Not elevated in normal pregnancy } \\
\text { May be elevated in pre-eclampsia, PE, } \\
\text { myocarditis, arrhythmias, sepsis }\end{array}$ \\
\hline D-dimer & $\begin{array}{l}\text { Use not recommended in pregnancy at } \\
\text { present }\end{array}$ \\
\hline CK & Normal range 5-40 IU/L ie lower in pregnancy \\
\hline Cholesterol & Elevated in pregnancy (do not check routinely) \\
\hline $\mathrm{TFTs}^{4}$ & Use gestation specific reference range \\
\hline ECG & $\begin{array}{l}\text { Sinus tachycardia } \\
15 \text { degree left axis deviation due to } \\
\text { diaphragmatic elevation } \\
\text { T wave changes - commonly t wave inversion } \\
\text { III and aVF } \\
\text { Non-specific ST changes, eg depression } \\
\text { Small Q waves }\end{array}$ \\
\hline Holter monitor & Supraventricular and ventricular ectopic beats \\
\hline CXR & $\begin{array}{l}\text { Prominent vascular markings } \\
\text { Raised diaphragm due to gravid uterus } \\
\text { Flattened left hemidiaphragm }\end{array}$ \\
\hline$A B G$ & Compensated metabolic alkalosis \\
\hline PEFR & Unchanged in pregnancy \\
\hline ECG & $\begin{array}{l}\text { All internal dimensions are mildly increased in } \\
\text { pregnancy }\end{array}$ \\
\hline CTPA & $\begin{array}{l}\text { Higher rate of non-diagnostic scans in } \\
\text { pregnancy }\end{array}$ \\
\hline $\begin{array}{l}\mathrm{V} / \mathrm{Q} \text { and } \mathrm{V} / \mathrm{Q} \\
\text { SPECT }\end{array}$ & $\begin{array}{l}\text { Interpretation comparable with outside of } \\
\text { pregnancy }\end{array}$ \\
\hline
\end{tabular}

$\mathrm{ABG}=$ arterial blood gas; $\mathrm{CK}=$ creatine kinase; $\mathrm{CTPA}=$ computerised tomography pulmonary angiogram; $C X R=$ chest $X$-ray; $E C G=$

electrocardiogram; $\mathrm{FBC}$ = full blood count; $\mathrm{Hb}=$ haemoglobin; $\mathrm{PE}=$ pulmonary embolism; PEFR = peak expiratory flow rate; $\mathrm{SPECT}=$ single-photon emission computed tomography; TFT = thyroid function tests; $\mathrm{WBC}=$ white blood cells.

mechanical effect of the enlarging uterus. ${ }^{10}$ If symptoms are associated with vomiting or abdominal tenderness, then the diagnosis should be questioned. Treatment with antacids, $\mathrm{H}_{2}$-receptor antagonists and proton pump inhibitors is safe in pregnancy, following lifestyle advice.

Pneumothorax can occur at any gestation, but should be considered as a cause of chest pain following a vaginal delivery. Management is the same as outside pregnancy. 
Cardiac disease is the single most common cause of maternal death in the UK and a high index of suspicion is needed. ${ }^{1}$ The incidence of acquired heart disease is increasing due to older age at first pregnancy and a higher prevalence of cardiovascular risk factors, such as hypertension, diabetes and obesity. ${ }^{11}$ Acute coronary syndromes are predominantly due to atherosclerotic disease, but there is an increased incidence of coronary artery dissection and thrombosis compared with the non-pregnant population. Percutaneous coronary intervention should not be withheld in pregnancy.

Peripartum cardiomyopathy (PPCM) is an idiopathic cardiomyopathy presenting with heart failure secondary to left ventricular systolic dysfunction (left ventricular ejection fraction $<45 \%$ ) towards the end of pregnancy or in the months following delivery, where no other cause of heart failure is found. ${ }^{12}$ Ejection fraction is less than $45 \%$ and no alternative cause of cardiomyopathy is identified. PPCM is more prevalent in women who are older, multiparous, hypertensive and Afro-Caribbean. The mainstay of treatment for PPCM is conventional pharmacological therapy for heart failure, such as diuretics, vasodilators and beta-blockers, and delivery of the baby. Anticoagulation is given to women with severely impaired left ventricular function, and some require antiarrhythmics. In the postpartum period, angiotensin-converting enzyme inhibitors are given. Many women recover within three to six months of diagnosis, but the prognosis is variable.

Aortic dissection is characterised by severe 'tearing' chest pain, often associated with systolic hypertension. This can be diagnosed with a chest CT scan.

\section{Palpitations}

Pregnant women may become more aware of their own heart beating in pregnancy due to sinus tachycardia and increased cardiac output. An increase in pulse rate of 10-20 bpm, particularly by the third trimester, is within normal limits. Women may experience a 'thump' from normal beats after a compensatory pause that follows ventricular ectopics. ${ }^{13}$ Those who report persistent or severe symptoms should be investigated.

The approach to identifying arrhythmias in pregnancy is to correctly diagnose the arrhythmia, check for underlying heart disease associated with the arrhythmia, then exclude systemic disease. ${ }^{14}$ If a woman with palpitations has a history or examination suggesting previous heart surgery, family history of sudden cardiac death or valvular heart disease, then this places her in a higher risk group.

Supraventricular tachycardias are common. Management is the same as outside of pregnancy, and vagotonic manoevres and adenosine can be used to reveal the underlying rhythm. Calcium channel blockers, such as verapamil and beta-blockers, are safe. Direct-current cardioversion can be performed with fetal monitoring and obstetric anaesthetic input.

Thyrotoxicosis should be excluded as a cause of tachycardia, because untreated hyperthyroidism can cause fetal complications, such as growth restriction, prematurity and stillbirth, as well as maternal complications of preeclampsia, congestive cardiac failure and thyroid storm. ${ }^{15}$ When interpreting thyroid function tests, clinicians should use trimester-specific reference ranges (see Table 1). Severe hyperemesis gravidarum (HG) can cause transient biochemical hyperthyroidism due to the thyroid-stimulating hormone (TSH)-like effects of the beta subunits of human chorionic gonadotropin. This occurs in over $60 \%$ of pregnancies with severe HG. Differentiation from first presentation of Graves' disease requires thorough clinical assessment. Negative TSHreceptor antibodies also support a diagnosis of HG.

Phaeochromocytoma should be considered in women with palpitations associated with severe atypical hypertension, headache, glucose intolerance and anxiety. A high index of suspicion is needed to make the diagnosis. Phaeochromocytoma is associated with maternal mortality of up to $50 \%$ at the time of labour or induction of general anaesthesia, ${ }^{16}$ and fetal mortality of approximately $26 \%$ in undiagnosed cases and $11 \%$ in diagnosed cases. ${ }^{17}$ In the last MBRRACE triennium, there were two deaths caused by phaeochromocytoma. Investigation is the same as outside pregnancy.

\section{Conclusion}

Women with pre-existing medical conditions who become pregnant and those who develop medical complications in pregnancy require a coordinated approach involving senior physicians and obstetricians. Physicians are experienced in assessing patients with chest pain, palpitations and/or breathlessness, and can make a valuable contribution to the care of pregnant women presenting with them provided they avoid errors of omission.

\section{References}

1 Knight M, Kenyon S, Brocklehurst P et al. Saving lives, improving mothers' care: lessons learned to inform future maternity care from the UK and Ireland confidential enquiries into maternal deaths and morbidity 2009-2012. Oxford: National Perinatal Epidemiology Unit, University of Oxford, 2014.

2 Nelson-Piercy C. Handbook of obstetric medicine, 5th edition. Boca Raton, FL: CRC Press, 2015.

3 Adamson D, Dhanjal M, Nelson-Piercy C. Heart disease in pregnancy. Oxford: Oxford University Press, 2011.

4 Cotzias C, Wong SJ, Taylor C et al. A study to establish gestationspecific reference intervals for thyroid function tests in normal singleton pregnancy. Eur J Obstet Gynecol Reprod Biol 2008;137:61-6.

5 British Thoracic Society/Scottish Intercollegiate Guidelines Network. SIGN 141. British guideline on the management of asthma. Edinburgh: Health Improvement Scotland, 2014.

6 Royal College of Obstetricians and Gynaecologists. Thromboembolic disease in pregnancy and the puerperium: acute management. Green Top Guideline. London: RCOG, April 2015.

7 Goodacre S, Nelson-Piercy C, Hunt B et al. When should we use diagnostic imaging to investigate for pulmonary embolism in pregnant and postpartum women? Emerg Med J 2015;32:78-82.

8 Ahearn GS, Hadjiliadis D, Govert JA et al. Massive pulmonary embolism during pregnancy successfully treated with recombinant tissue plasminogen activator. Arch Int Med 2002;162:1221-7.

9 Siscione AC, Ivester T, Largoza M et al. Acute pulmonary oedema in pregnancy. Obstet Gynaecol 2003;101:511-5.

10 Richter JE. Gastroesophageal reflux disease during pregnancy. Gastroenterol Clin North Am 2003;32:235-61.

11 Nanda S, Nelson-Piercy C, Mackillop L. Cardiac disease in pregnancy. Clin Med 2012;12:553-60. 
12 Sliwa K, Hilfiker-Kleiner D, Petrie MC et al. Current state of knowledge on aetiology, diagnosis, management, and therapy of peripartum cardiomyopathy: a position statement from the Heart Failure Association of the European Society of Cardiology Working Group on peripartum cardiomyopathy. Eur J Heart Fail 2010; 12:767-78.

13 Middleton D, Aljuboori S, Banerjee A et al. Problem based review: the pregnant woman presenting to the amu with palpitations. Acute Med 2014;13:139-42.

14 Adamson D, Nelson-Piercy C. Managing palpitations and arrhythmias during pregnancy. Heart 2007;93:1630-6.
15 Girling J. Thyroid disease in pregnancy. TOG 2008;10;237-43.

16 Lau P, Permezel M, Dawson P et al. Phaeochromocytoma in pregnancy. Aust N Z J Obstet Gynaecol 1996;36:472-6.

17 Frise CJ, Williamson C. Endocrine disease in pregnancy. Clin Med 2013;13:176-81.

Address for correspondence: Prof C Nelson-Piercy, 10th Floor Directorate Office, North Wing, St Thomas' Hospital, Guy's and St Thomas' NHS Foundation Trust, Westminster Bridge Road, London SE1 7EH, UK.

Email: catherine.nelson-piercy@gstt.nhs.uk

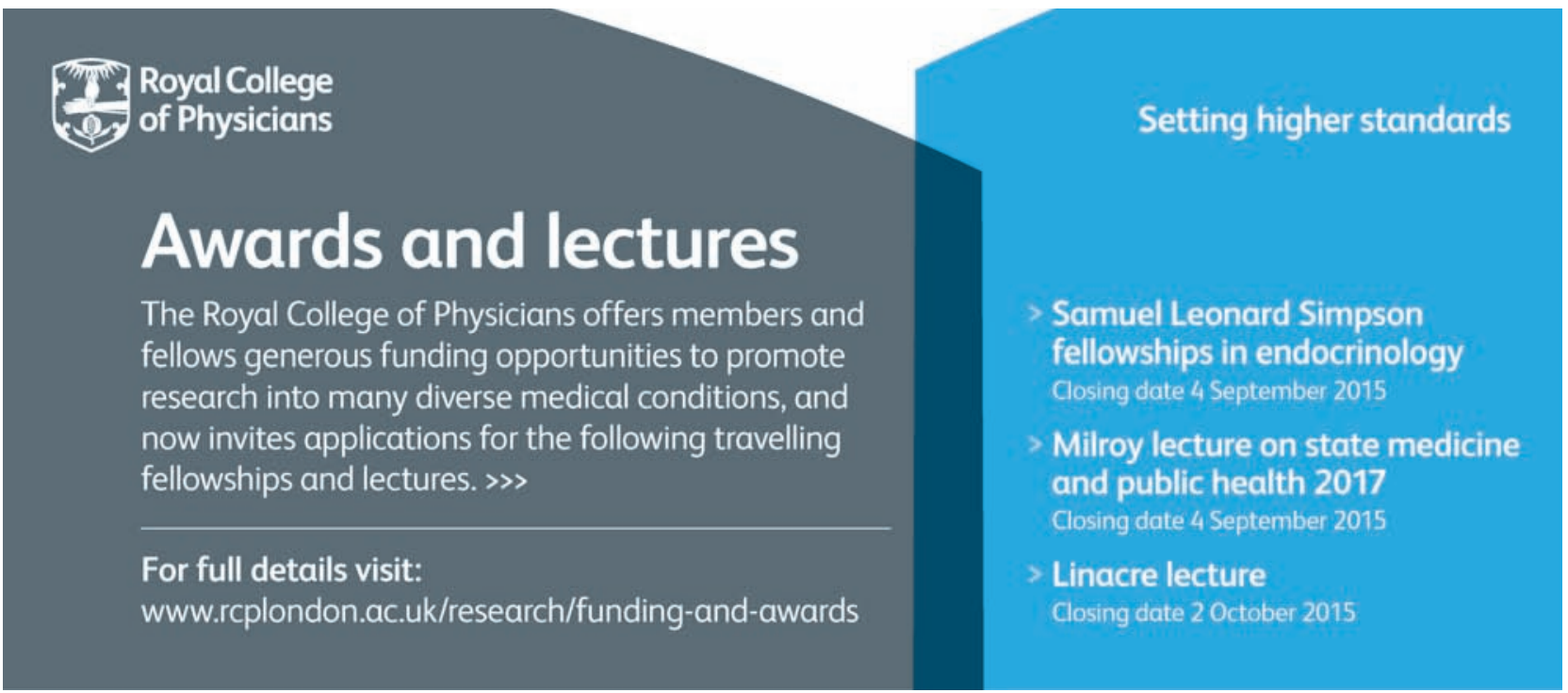

